\title{
Special feature: Lung injury
}

\section{Review \\ Computational modelling of lung injury: is there potential for benefit?}

\author{
Daniel J. R. Harvey and Jonathan G. Hardman* \\ Division of Anaesthesia and Intensive Care, University of Nottingham and Nottingham University \\ Hospitals NHS Trust, Nottingham, UK
}

\begin{abstract}
State-of-the-art medical care of the victims of current conflicts is generating large quantities of quality clinical data as a by-product. Observational research based on these data is beginning to have a profound influence on the clinical management of both military and civilian trauma patients. Computational modelling based on these datasets may offer the ability to investigate clinical treatment strategies that are practically, ethically or scientifically impossible to investigate on the front line. This article reviews the potential of this novel technology to aid development of treatment for blast lung and other unresolved medical scenarios.
\end{abstract}

Keywords: blast lung; respiratory failure; respiratory distress syndrome; model; computer simulation

\section{INTRODUCTION}

Many researchers feel that conducting even simple clinical trials can seem a Herculean task. The increasing bureaucracy of research governance, though of critical importance, tends to make the process slow, expensive and time consuming. Even more respect and admiration is therefore due to those who conduct clinical trials during the very real trials of battle. Outstanding efforts have recently been made by clinicians on the front lines in Afghanistan and Iraq into topics such as traumatic coagulopathy [1-2] and damage control surgery [3], and the important insights gained impact not only on future war casualties, but also on the victims of more mundane but equally fatal trauma closer to home [4].

The problems of conducting clinical trials in this environment are multiple. The military train of command does not extend to offering blanket ethical approval, and the concepts of informed consent and ethical oversight might seem very alien on the battlefield. Classical research methodology (for instance, randomization and blinding) can be very difficult or impossible. Patients have heterogeneous patterns of injury, and their numbers, although high in human terms, may offer limited statistical power. Indeed experimentation on the battlefield is prohibited by US federal law [5]. These difficulties lead to the evolution of practice based on retrospective, observational data empowered by the military's abilities to disseminate best practice protocols rapidly to the front line and ensure that they are followed. These observational and cohort data are frequently used to generate questions that can then be investigated using animal

* Author for correspondence.

One contribution of 20 to a Theme Issue 'Military medicine in the 21 st century: pushing the boundaries of combat casualty care'. models, the results feeding back into practice protocols amenable to repeated audit. While the evidence base is grade II or III at best [6], there seems no potential for true randomized controlled trials in this context.

\section{WHAT DOES MODELLING HAVE TO OFFER?}

Much scientific research is conducted using in silico modelling, the fields of climate research and economics being topical examples. While it would be foolish to claim to model reality in every detail, it is widely recognized that carefully developed, empirically based and validated computer models have much to offer. Models are in frequent use throughout medical research but are not always recognized as such; for example, many studies that utilize a correlation between surrogate and clinical outcomes rely on an underlying model, which relates the two. Likewise much statistical analysis used to evaluate the results is reliant on statistical models of data relationships.

Modelling is the reproduction of real-life shape, form or function [7-8]. It can be physical, mental, computational, statistical (probabilistic), mathematical, animal or laboratory. It is an attempt to simulate or recreate the important and interesting aspects of a question or system, while excluding the unpredictable and irrelevant aspects of organisms and populations that add heterogeneity, random variation and pragmatic obstructions. Such modelling may allow us to elucidate complex issues or to formalize or automate decision-making processes with a view to treating patients $[9-12]$. The valuable efforts of scientists in the battlefield can provide the empirical data on which a model can be built. Treatment strategies can be investigated in an in silico model rapidly and to a level of complexity not possible in animal models [13-14]. Even where there are gaps in the 


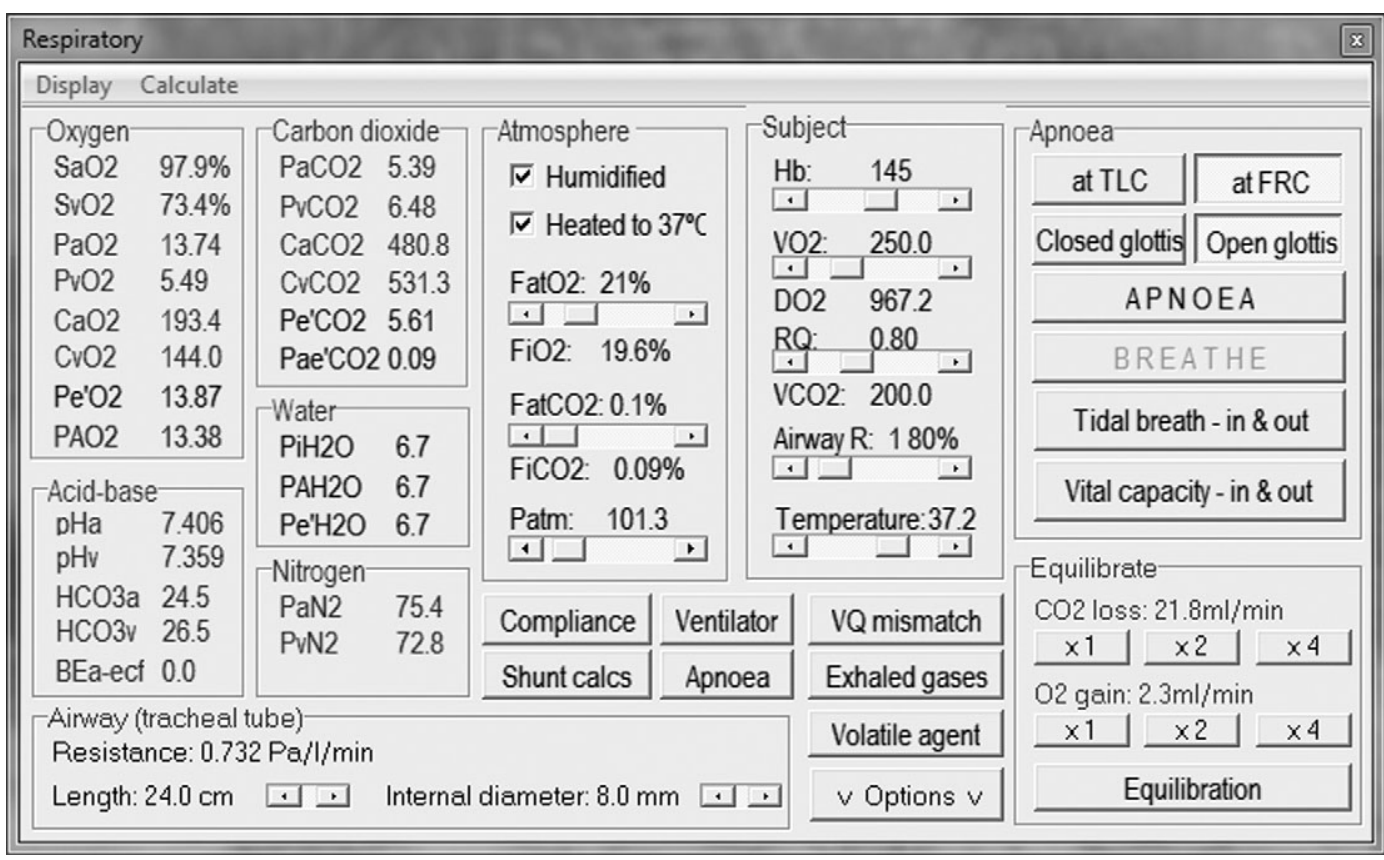

Figure 1. Respiratory physiological variable control panel.

knowledge needed to construct a model, it is still possible to gain valid results provided the gaps are known and the resulting limitations acknowledged. A credible in silico model might best be considered akin to an early Petri dish investigation: an efficient, rapid and cheap method of rejecting grossly ineffective strategies and selecting those ripe for further investigation, perhaps in other models or direct to patient cohorts where the validity is strong. Indeed the concept of Monte Carlo testing of many subtly different protocols in an established model is a powerful one [15], and is perhaps the only method of selecting the 'best-fit' algorithm with a high level of fidelity. Furthermore, in silico modelling allows investigation of physiological situations that are impossible or unethical to reproduce in a controlled way in human studies, but which are, nonetheless, credible reflections of existing pathologies [16-17].

Our own respiratory computational model (the Nottingham physiology simulator) integrates respiratory physiology with mechanical ventilation, allowing subtle and precise alteration of more than 50 variables (figures 1-3). For instance, compliance and resistance to both gas and vascular flow to 100 representative alveolar units can be adjusted to mimic known pathological conditions. Downstream tissue perfusion outcome measures (such as arterial gas partial pressures) can be calculated after exposure to a myriad of ventilatory strategies. This model has been successfully deployed in characterizing deterioration in tissue oxygenation during apnoeic episodes, aiding our understanding of management of airway emergencies [16].

What are the questions confronting clinicians at the front that might be illuminated by the application of in silico modelling? The incidence of blast lung appears to be increasing owing to increases in the power of improvised explosive devices and their use in confined spaces [18-19]. The relative infrequency of blast lung [20], its geographical dispersion and the obvious chaos of the initial insult make traditional investigation virtually impossible for the reasons described above. There is an ethical obligation to minimize the number of animals used in research, although animal-derived data are likely to be a very important aspect of model development and subsequent validation. Blast lung's importance to the field is not doubted; it is the commonest cause of death in initial survivors of explosions [18]. Animal models suggest that relatively high pressures are required to trigger blast lung [21] and that victims exposed to real world pressure waves of this magnitude are often sufficiently close to the explosion for fragmentation injuries to predominate [22].

The ability to model individual lung units down to the alveolar level facilitates the study of pathologies whose distribution within the organ are heterogeneous (for instance, adult respiratory distress syndromeARDS), and our model has been validated for studying this condition [23]. Heterogeneous lung damage is also seen in blast lung where perihilar infiltrates are virtually pathognomonic [24]. Data extrapolated from ARDS patients have been used to guide therapy in these patients, although the pathophysiology, distribution of injury and progression may be quite different [25]. Thus, most centres have used a lowvolume, pressure-limited strategy resorting to rescue therapies such as nitric oxide and ECMO when necessary [18] in blast lung. A validated model based on an empirical dataset gained in the field, and cross-validated against the same patient cohorts, animal models and laboratory models would allow the detailed investigation of unanswered questions; for example, the role of PEEP and the potential advantages of high-frequency oscillatory ventilation. In addition, an unlimited number of clinical scenarios can be investigated to suggest important contributory factors in poor outcome (e.g. massive haemorrhage, periods of hypotension or apnoea).

There is a substantial body of model evidence for physical patterns of blast injury, which has been 


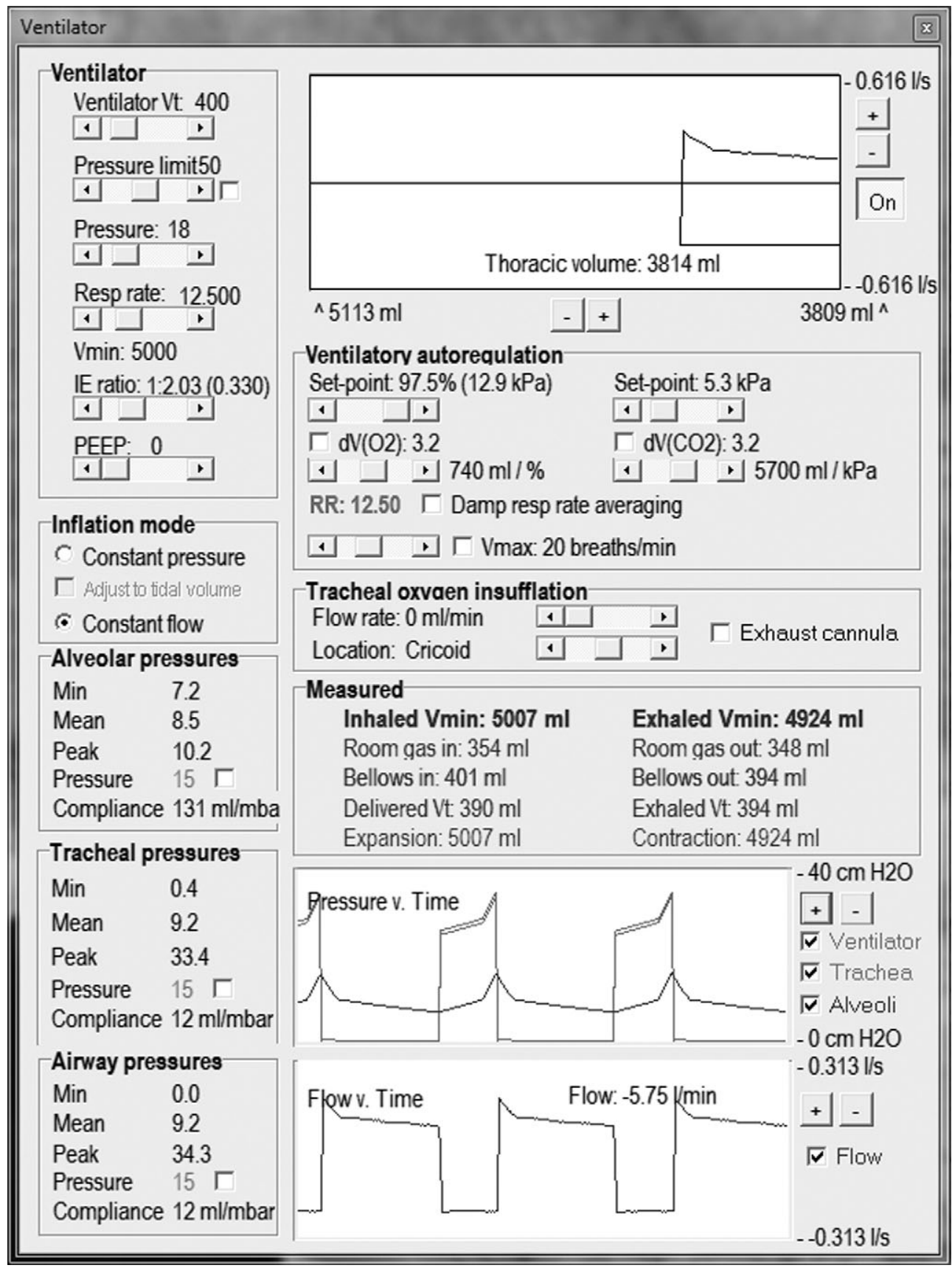

Figure 2. Ventilator control panel.

undertaken both in the laboratory [26-28] and in silico [29-31] that could inform a preliminary physiological model. Indeed computational modelling was identified as a potentially fruitful avenue for blast lung research by a consensus workshop and in a recent review [22].

Integrated respiratory and cardiovascular models bring with them the possibility of further refining military trauma protocols already being successfully deployed on the battlefield. Such a model would permit detailed investigation of the response of tissue perfusion parameters to almost infinite constellations of pathologies and severity, allowing the development of sophisticated and individualized therapies. For instance, severe burns coupled with lung contusions and major haemorrhage presents a complex challenge, and resuscitation goals in this situation are uncertain. Current available evidence shows that as well as the choice of intervention, its precise timing is critical
[32]. The value of the clinical acumen of experienced clinicians cannot be underestimated, but the ability to model responses to different resuscitation strategies may be very helpful (for instance, in rapidly determining the theoretical optimal timing and targets of cardiovascular resuscitation). Decisions regarding timing of risky procedures likely to worsen physiological status (for instance, global repatriation and surgical intervention) can be illuminated by computational models, which also allow for progression of underlying pathological insult, seen in conditions such as blast lung [25].

\section{PRINCIPLES OF MODELLING}

Modelling can be undertaken in a 'top-down' or 'bottom-up' methodology. The top-down approach involves construction of a model that appears correct 


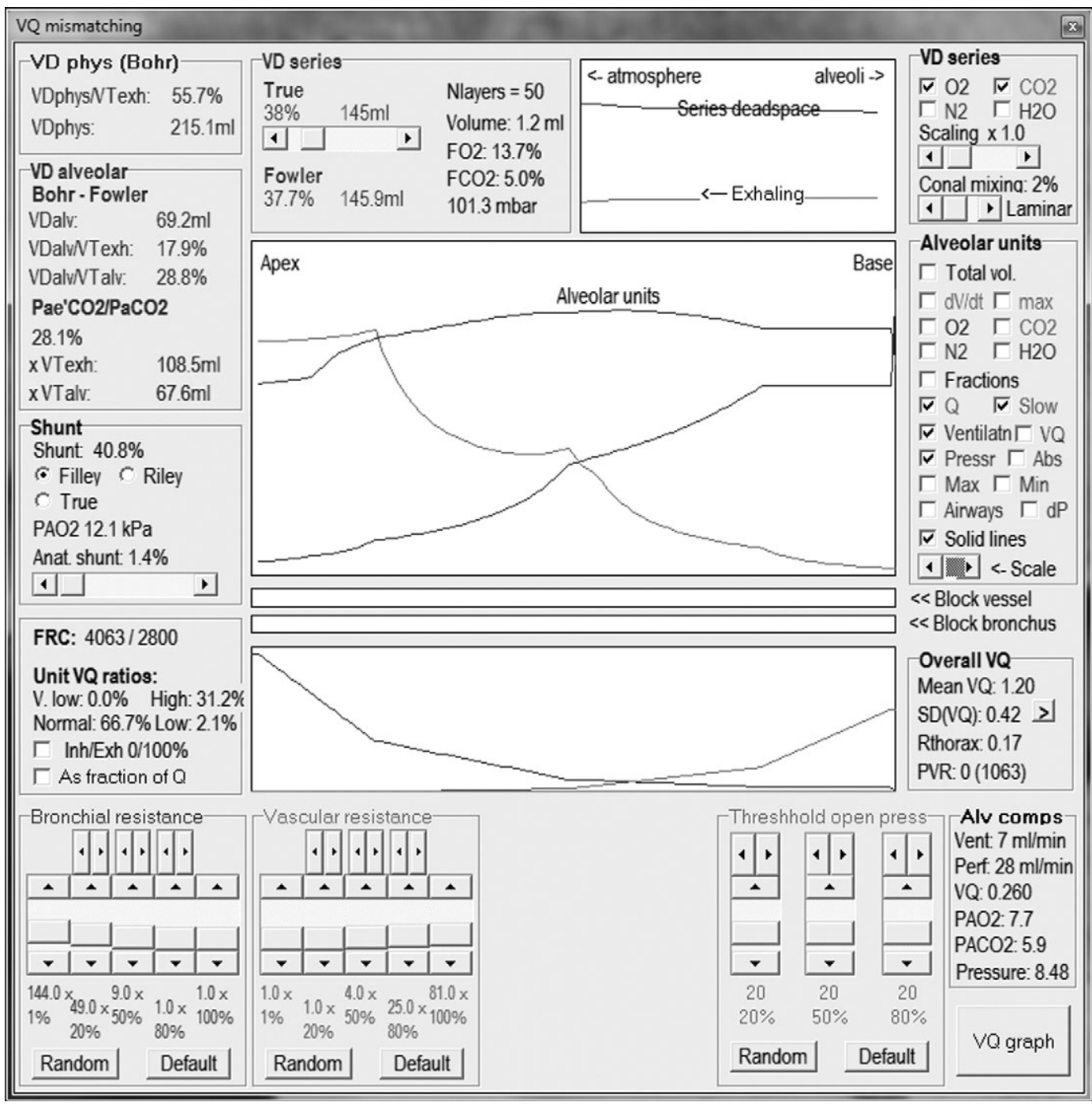

Figure 3. VQ mismatching control panel.

and then entering a process of validation against datasets with subsequent refinement and repeat investigation, building the 'machinery' of the model after the initial credible output is established. Bottom-up construction is scientifically more robust, involving building of a model from first physiological principles, but is slower and requires data that may not exist or may be extremely difficult to collect. The end result of both processes may be a model that looks remarkably similar; in any case, a key concept within modelling is that the model does not represent truth, but is close enough to it for practical purposes (in that no model is valid, merely fit for purpose). Once the model has been developed, validation against existing human and animal datasets, or if possible, prospective comparison against real-time patient cohorts will provide credibility. Investigation of treatment strategies can be begun in earnest at this stage, although the model should be continually developed in the light of emerging knowledge.

Computational modelling is not a panacea; indeed the potential problems in generating a credible model of blast lung can be readily seen. A human dataset will be required for model development and validation, and this will be challenging to acquire. However, the establishment of military/civilian research partnerships (for instance, the US Institute of Surgical Research collaboration with the 31st Combat Support Hospital and the Royal Centre for Defence Medicine in the UK) will facilitate this process. Furthermore, modelling is not a resource-free exercise; large amounts of computational power are required and model development can be very labour intensive. Additionally, model development can often be hindered by the absence of a few relatively minor but critical aspects of knowledge. Treatment algorithms and physiological insights gained from computational modelling must be regarded as theoretical until confirmed in human cohorts, although this need not prevent incorporation into front-line practices where no conflicting evidence exists.

\section{CONCLUSIONS}

Military and civilian victims of war and terrorism have different pathologies and patterns of injury compared with those suffering civilian trauma. These patient cohorts have been very difficult to study historically but there is an increasing quantity and quality of data emanating from Iraq, Afghanistan and other military arenas. The human price that has been paid to make these data available makes the imaginative and 
efficient use of these data a moral and practical imperative. The onus is on the scientific community to maximize the benefit derived by future victims, and computational modelling may well have a valuable role to play. The current development of clinical care on the battlefield by meticulous observational data collection and synthesis with current understanding may be considerably quickened by the application of systemic pathophysiological investigation with appropriately validated computational models. Furthermore, the data derived from animal models will accelerate the synthesis of these data into credible models, allowing us to truly translate basic science research into battlefield medicine. Modelling will allow us to open a conduit from human research (with its noisy data), animal research (with its limited mapping to human pathophysiology) to the development of real-world clinical treatment strategies; modelling aggregates our accumulated knowledge, expanding the importance and relevance of previous work, drawing together ideas and data from disparate sources and groups and forging a clear highway from isolated knowledge to human clinical practice.

\section{REFERENCES}

1 Spinella, P. C. 2008 Warm fresh whole blood transfusion for severe hemorrhage: US military and potential civilian applications. Crit. Care Med. 36(Suppl. 7), S340-S345.

2 Spinella, P. C. et al. 2008 The effect of recombinant activated factor VII on mortality in combat-related casualties with severe trauma and massive transfusion. F. Trauma 64, 286-293. (doi:10.1097/TA.0b013e318162759f)

3 Beekley, A. C. 2008 Damage control resuscitation: a sensible approach to the exsanguinating surgical patient. Crit. Care Med. 36(Suppl. 7), S267-S274.

4 Holcomb, J. B. et al. 2008 Increased plasma and platelet to red blood cell ratios improves outcome in 466 massively transfused civilian trauma patients. Ann. Surg. 248, 447-458.

5 Grathwohl, K. W., Venticinque, S. G., Blackbourne, L. H. \& Jenkins, D. H. 2008 The evolution of military trauma and critical care medicine: applications for civilian medical care systems. Crit. Care Med. 36, S253-S254.

6 Guyatt, G. H., Oxman, A. D., Vist, G. E., Kunz, R., Falck-Ytter, Y., Alonso-Coello, P., Schünemann, H. J. \& GRADE Working Group. 2008 GRADE: an emerging consensus on rating quality of evidence and strength of recommendations. BMF (Clinical Research ed.) 336, 924-926. (doi:10.1136/bmj.39489.470347.AD)

7 Masuzawa, T., Fukui, Y. \& Smith, N. T. 1992 Cardiovascular simulation using a multiple modeling method on a digital computer - simulation of interaction between the cardiovascular system and angiotensin II. $\mathcal{F}$. Clin. Monit. 8, 50-58. (doi:10.1007/BF01618088)

8 Greenway, C. V. 1981 Mechanisms and quantitative assessment of drug effects on cardiac output with a new model of the circulation. Pharmacol. Rev. 33, 213-251.

9 Kwok, H. F., Linkens, D. A., Mahfouf, M. \& Mills, G. H. 2003 Rule-base derivation for intensive care ventilator control using ANFIS. Artif. Intell. Med. 29, 185-201. (doi:10.1016/S0933-3657(02)00074-X)

$10 \mathrm{Lau}$, F. 1994 A clinical decision support system prototype for cardiovascular intensive care. Int. F. Clin.
Monit. Comput. 11, 157-169. (doi:10.1007/BF011 32364)

11 Hoeksel, S. A., Blom, J. A., Jansen, J. R., Maessen, J. G. \& Schreuder, J. J. 1999 Automated infusion of vasoactive and inotropic drugs to control arterial and pulmonary pressures during cardiac surgery. Crit. Care Med. 27, 2792-2798. (doi:10.1097/00003246-199912000-00031)

12 Bates, J. H. \& Young, M. P. 2003 Applying fuzzy logic to medical decision making in the intensive care unit. Am. F. Respir. Crit. Care Med. 167, 948-952. (doi:10. 1164/rccm.200207-777CP)

13 Hardman, J. G. \& Wills, J. S. 2006 The development of hypoxaemia during apnoea in children: a computational modelling investigation. Br. F. Anaesth. 97, 564-570. (doi:10.1093/bja/ael178)

14 Kathirgamanathan, A., McCahon, R. A. \& Hardman, J. G. 2009 Indices of pulmonary oxygenation in pathological lung states: an investigation using high-fidelity, computational modelling. Br. F. Anaesth. 103, 291297. (doi:10.1093/bja/aep140)

15 Babbs, C. F. \& Kern, K. B. 2002 Optimum compression to ventilation ratios in CPR under realistic, practical conditions: a physiological and mathematical analysis. Resuscitation 54, 147-157. (doi:10.1016/S03009572(02)00054-0)

16 Hardman, J. G., Wills, J. S. \& Aitkenhead, A. R. 2000 Factors determining the onset and course of hypoxemia during apnea: an investigation using physiological modelling. Anesth. Analg. 90, 619-624. (doi:10.1097/ 00000539-200003000-00022)

17 McNamara, M. J. \& Hardman, J. G. 2005 Hypoxaemia during open-airway apnoea: a computational modelling analysis. Anaesthesia 60, 741-746. (doi:10.1111/j.13652044.2005.04228.x)

18 Ritenour, A. E. \& Baskin, T. W. 2008 Primary blast injury: update on diagnosis and treatment. Crit. Care Med. 36(Suppl. 7), S311-S317.

19 Langworthy, M. J., Sabra, J. \& Gould, M. 2004 Terrorism and blast phenomena: lessons learned from the attack on the USS Cole (DDG67). Clin. Orthopaed. Relat. Res. 422, 82-87. (doi:10.1097/01.blo. 0000128293.43913.ca)

20 Smith, J. E. 2011 The epidemiology of blast lung injury during recent military conflicts: a retrospective database review of cases presenting to deployed military hospitals, 2003-2009. Phil. Trans. R. Soc. B 366, 291-294. (doi:10.1098/rstb.2010.0251)

21 Chavko, M., Prusaczyk, W. K. \& McCarron, R. M. 2006 Lung injury and recovery after exposure to blast overpressure. F. Trauma 61, 933-942. (doi:10.1097/01.ta. $0000233742.75450 .47)$

22 Champion, H. R., Holcomb, J. B. \& Young, L. A. 2009 Injuries from explosions: physics, biophysics, pathology, and required research focus. F. Trauma 66, 1468-1477. (doi:10.1097/TA.0b013e3181a27e7f)

23 McCahon, R. A., Columb, M. O., Mahajan, R. P. \& Hardman, J. G. 2008 Validation and application of a high-fidelity, computational model of acute respiratory distress syndrome to the examination of the indices of oxygenation at constant lung-state. Br. F. Anaesth. 101, 358-365. (doi:10.1093/bja/aen181)

24 Avidan, V., Hersch, M., Armon, Y., Spira, R., Aharoni, D., Reissman, P. \& Schecter, W. P. 2005 Blast lung injury: clinical manifestations, treatment, and outcome. Am. F. Surg. 190, 945-950. (doi:10.1016/j.amjsurg. 2005.08.022)

25 Elsayed, N. M. \& Gorbunov, N. V. 2007 Pulmonary biochemical and histological alterations after repeated low-level blast overpressure exposures. Toxicol. Sci. 95, 289-296. 
26 Jaffin, J. H. et al. 1987 A laboratory model for studying blast overpressure injury. F. Trauma 27, 349-356. (doi:10.1097/00005373-198704000-00002)

27 Stuhmiller, J. H. 1997 Biological response to blast overpressure: a summary of modeling. Toxicology 121, 91103. (doi:10.1016/S0300-483X(97)03658-5)

28 Hayda, R., Harris, R. M. \& Bass, C. D. 2004 Blast injury research: modeling injury effects of landmines, bullets, and bombs. Clin. Orthopaed. Relat. Res. 422 97-108. (doi:10.1097/01.blo.0000128295.28666.ee)

29 Taylor, P. A. \& Ford, C. C. 2009 Simulation of blastinduced early-time intracranial wave physics leading to traumatic brain injury. F. Biomech. Eng. 131, 061007. (doi:10.1115/1.3118765)

30 Moore, D. F., Jérusalem, A., Nyein, M., Noels, L., Jaffee, M. S. \& Radovitzky, R. A. 2009 Computational biology-modeling of primary blast effects on the central nervous system. NeuroImage 47(Suppl. 2), T10-T20.

31 Stuhmiller, J. H., Chuong, C. J., Phillips, Y. Y. \& Dodd, K. T. 1988 Computer modeling of thoracic response to blast. F. Trauma 28(Suppl. 1), S132-S139.

32 Blackbourne, L. H. 2008 Combat damage control surgery. Crit. Care Med. 36(Suppl. 7), S304-S310. 